\title{
Switch operation safety
}

\author{
Boris Gluzberg ${ }^{1}$, Vadim Korolev $^{2}$, Alexey Loktev ${ }^{2}$, Irina Shishkina, ${ }^{2, *}$ and Mikhail \\ Berezovsky ${ }^{3}$ \\ ${ }^{1}$ Joint Stock Company "Scientific Research Institute of Railway Transport", 3rd \\ Mytishchinskaya st., 10, 129626, Moscow, Russia \\ ${ }^{2}$ Russian University of Transport (RUT - MIIT)), Chasovaya St., 22/2, 125190, Moscow, \\ Russia \\ ${ }^{3}$ JSC "Novosibirsk switch plant", Aksenova str., 7, 630025, Novosibirsk, Russia
}

\begin{abstract}
The article studied the safety of passing the rolling stock by railway switches. On railway switches, counter rails provide the safe passage of the rolling stock by the wheelsets through the harmful space of the cross. The levels of dynamic effects arising from the passage of carriages by the railway switch significantly depend on the relative position of the wheelsets and elements of the switch, which is determined by the combination of their main geometric dimensions. To calculate the most unfavorable combinations of wheelset and gauge dimensions, taking into account the tolerances adopted for these dimensions, the method of probability compositions is used, based on the application of the theory of probability. The calculating apparatus of the approach using conditional probabilities of events based on the formulas of total probability and Bayes formula. The level of impact of the wheels on the counter-rail is regulated by rationing the values of the "impact effect" on the bent and catching parts of the counter-rail.
\end{abstract}

\section{Introduction}

On railway switches, the safe passage of the wheelsets of the rolling stock through the harmful space of the cross is provided by counter-rails [1-3].

The first criterion of importance is traffic safety. The parameters of the counter-rail and counter-rail angle as a whole should ensure that wheelsets pass without hits and wheel overrunning on the tip of the cross core, on the "non-working" guardrail to the frog throat, on the catching part of the guardrail, catching part of the counter-rail itself, to prevent the wheelset from spreading between the counter-rail and "non-working" guard-rail. Also undesirable are the wheels overrunning on the "non-working" guardrail between the throat of the cross and its point.

The second most important criterion is a dynamic kinematic criterion. The limitation of the effect of rolling stock wheels on the counter-rail unit. The semantic meaning of this criterion is the prevention of abrupt shifts of wheel sets as they pass through the crossbar zone, splitting and destruction of the counter-rail node, its rapid breakdowns in operation.

\footnotetext{
* Corresponding author: shishkinaira@inbox.ru
} 
Its quantitative expression is a parameter associated with the loss of kinetic energy when a wheel hits or overruns a counter rail. [4-6]

The third criterion is to ensure the strength of the counter rail itself. The impact of the wheels on the counter rail should not lead to the appearance of dangerous defects or kinks of the counter rail. The criterion is checked by comparing the stresses arising in the most loaded part of the counter rail with the permissible stresses.

The criteria listed above are necessary and sufficient requirements, based on which regulatory requirements for the counter-rails deterioration and the size of their grooves should be formed.

\section{Research Methods}

The levels of dynamic effects that occur during the passage of carriages by the rail-way switch significantly depend on the relative position of the wheelsets and elements of the switch, which is determined by the combination of their basic geometric dimensions [7].

The simplest method (limit combinations method) consists in calculating the most unfavorable combinations of wheelsets and wheel gauge dimensions, taking into account the tolerances adopted for these dimensions. Unfortunately, for all the simplicity and clarity of this method, it contains contradictions that make the calculations performed with its help conditional. For example, as shown by mass measurements of wheelsets, the minimum sum of the thickness of two ridges of one wheel pair in operation cannot be equal to the sum of the two minimum permissible thicknesses of the ridges.

To eliminate the contradictions of the method of limiting combinations, a method of probabilistic compositions was proposed based on the application of the theory of probability. The limiting ratios in this method are estimated by values that have a probability of violation of ratios not more than once per set period (service life).

The method works well in analyzing the conditions for the passage of "all" wheel-sets for "all" railway switches, but does not allow to consider the passage of "all" wheelsets for a switch with specifically specified dimensions.

In order leaving the need to use this and other assumptions is possible if, retaining the idea of a probabilistic approach to considering the movement of a wheelset by a cross-joint proceed from composing dimensions to calculation using conditional probabilities of events. Such an approach makes it possible to calculate the phenomena probabilities for specific combinations of gauges and gutters.

The calculating apparatus of the approach using conditional probabilities of events uses the formulas of total probability and Bayes formulas.

$$
\phi\left(X_{1} ; X_{2}\right)=\phi_{1}\left(X_{1}\right) \cdot \phi_{2 \mid 1}\left(X_{2} \mid X_{1}\right)
$$

where: $X_{1}$ and $X_{2}$ - considered events;

$\varphi 1$ is the probability distribution density of the X1 event;

$\varphi$ is the distribution density of the simultaneous event $\mathrm{X} 1$ and $\mathrm{X} 2$;

$\varphi 2 \mid 1$ is the conditional probability of the $\mathrm{X} 2$ event at the condition, that the $\mathrm{X} 1$ event has occurred.

This approach was used in the present work.

The main initial calculation data is the position of the wheel in the track at the entrance to the joint, which is determined by the gap between the wheel and the rail element of the joint. When calculating for a cross-joint with clearly specified dimensions, the distribution of the total gap of the wheelset in the track ( $\delta$ max) can be obtained as follows:

$$
P\left\{\delta_{\max }\right\}=P\left\{\left[S+S^{y}(S)\right]-\left[\left(Q-Q^{y}\right)+D_{k}+D_{y}\right]\right\}
$$


where: $P$ - corresponding event probability;

$S$, and $S^{y}$ - track and its elastic deformation, respectively;

$Q$ and $Q^{y}$ - distance between the wheel flanges at the measurement of the track width and its elastic change;

$D_{k}$ and $D^{y}$ - thicknesses of the wheelset wheel flanges from the counter-rail and the guard-rail.

Distribution of a complex of dimensions related to the track can be obtained using the experimental dependence of the elastic deformations of the track on its size, obtained by direct tests:

$$
\varphi_{\Sigma}\left(S_{\Sigma}\right)=\varphi_{\left(S+S^{y}\right)}\left[\left(S+S^{y}(S)\right) \mid S\right]=\varphi_{S^{y}}\left(S_{\Sigma}-S\right)
$$

where $\mathrm{S} \Sigma$ is the width of the gauge taking into account the elastic deformations corresponding to a given static gauge width.

It is advisable to take the distribution of the complex of dimensions related to wheelsets directly based on the results of measurements.

Within the gap $\delta$ max, different positions of the wheelset are possible. Their probability is determined by the wagging waveform and the moment the wheel pair enters the cross joint.

In straight sections of the track, wagging of carriages is close to sinusoidal. By simple calculations, it can be obtained that with a sinusoidal wagging waveform, the probability density $\delta$ can be determined by the formula:

$$
\phi\left(\delta \mid \delta_{\max }\right)=\frac{2}{\pi \cdot \delta_{\max }} \cdot \frac{1}{\sqrt{\left(\delta_{\max }-\delta\right) \cdot \delta}}
$$

The obtained distributions $\delta \max$ can be used to calculate the distribution of wheel positions relative to the rail using conditional probabilities:

$$
\begin{gathered}
p\left\{\delta, \delta_{\max }\right\}=p\left\{\delta_{\max }\right\} \cdot p\left\{\delta \mid \delta_{\max }\right\} \\
P_{\delta}=\int_{\delta_{\max }} p\left\{\delta, \delta_{\max }\right\} d \delta_{\max }
\end{gathered}
$$

(In expression (6) and further in expressions for probabilities we mean the Lebesgue integral).

Calculations should be made for each specific value of the gauge width taking into account the elastic deformations that occur with a given gauge width taken from the test results.

By summing up the distribution of the considered gaps within all thickness of wheel flanges, we obtain the distribution of the distance between the lateral working edge of the rail and the non-working edge of the wheel $\delta_{D}$ :

$$
\phi_{\delta_{D}}\left(\delta_{D}\right)=\int_{\delta_{D}} \phi_{\delta}(\delta) \cdot \phi_{D}\left(\delta_{D}-\delta\right) d \delta_{D}
$$

From this distribution, within the specific dimensions of the gutters of the counter-rails, it is possible to determine the probabilities of wheel hits to the catching part of the counter- 
rails and the overrunning on its bent part for each specific size of the gutters of the counter rail in its corresponding part for a specifically given gauge:

$$
P_{o k}=\int_{e_{k o}}^{e k} \phi\left(\delta_{D}\right) d \delta_{D} P_{o k}=\int_{e_{k o}}^{e k} \phi\left(\delta_{D}\right) d \delta_{D}
$$

where $\mathrm{P}_{\mathrm{pk}}, \mathrm{P}_{\mathrm{ok}}$ are, respectively, the probabilities of wheels overrunning to the catching part and the bent part of the counter-rail.

The position of the wheel in the gutter of the straight part of the counter-rail is determined for specific values of the gutters because of the conversion of distributions, the position of the wheelset in the rail track by the "retracting" action of the counter rail. In this case, it is necessary to take into account the connection between the position of the wheel relative to the counter-rail and the elastic deformation of the counter-rail itself.

Let us consider the appearance of the wheel in the straight part of the counter-rail with the overrunning on its bent part. Before the counter-rail touching the probability of the position of the rear part of the wheel flange has a distribution $\mathrm{P}\left(\delta_{D}\right)$. For those wheels for which $\delta_{D} \leq e_{k}$ the distribution of the gap between the running rail and the back of the flange after entering the straight part of the counter rail will remain unchanged, since they will not be in contact with the counter rail.

Wheels for which $\delta_{D}>e_{k}$ will be in contact with the counter-rail. While moving the wheel cause the elastic deformation of the counter-rail еку. The position of the wheel in the straight part of the counter rail will be determined by the magnitude of this elastic deformation.

If we use the experimentally obtained distributions of elastic squeezing of the counterrail at different values of the run of the wheels on the bent part, then we will have, passing to probability densities:

$$
\left\{\begin{array}{c}
\varphi_{\delta_{D}}\left(\delta_{D}\right) \quad \delta_{D} \leq e_{k} \\
\varphi_{\delta_{k}^{n}}\left(\delta_{k}^{n}\right)=\int_{\left(\delta_{D}-e_{k}\right)} \varphi_{\delta_{D}}\left(\delta_{D}\right) \cdot \varphi_{\left.e_{k}^{y}\right|_{3}}\left(\delta_{\kappa}^{2}-e_{k}\right) d \delta_{D} \delta_{D}>e_{k}
\end{array}\right.
$$

where: $\left.\quad e_{k}^{y}\right|_{3}$ - probability density of the elastic deformation of the counter-rail е ку during the overrunning of the wheel on the bent part.

The calculation is performed for each of the possible combinations of gauge dimensions and the counter- rail gutter.

Then, the compositions of the gap between the running rail and the wheel flange in the chute of the counter rail and the parameter (nozzle plus the sum of the wheelset flanges) are constructed in a similar way. The density of this distribution is determined by the probability of the wheels overrunning on the edge of the crossbar with the help of integration.

Convolutions of the distributions for calculating the probabilities of various phenomena within a certain range of changes in the dimensions of the grooves and the gauge, as well as for the conditions on the network, are made taking into account the conditional probabilities based on the actual size distributions of the cross-joint.

To obtain the numerical values of the cross-joints of the switches dimension's ratios, measurements were made on the main and receiving-departure routes. 
The distribution of the complex of dimensions related to the wheelsets is taken from the results of measurements carried out by the laboratory of the «Strelochnoye khozyaystvo» of «VNIIZhT» JSC. Measurements were performed at the Moscow unit of the Moscow Railway. According to the distributions of the dimensions of the cross-joints gauge and grooves obtained in measurements, the probabilities of the occurrence of adverse events during the passage of the cross-joints by the rolling stock with the specified combinations of the dimensions encountered in the practice of operation were calculated.

From these distributions, by calculating their densities and summing up within the specific dimensions of the counter-rail grooves, we can determine the probabilities of gaps between the back side of the wheel and the rail when entering the crossbar (figure 1) and the distances between the working side of the rail and the rear edge of the wheel (figure 2).

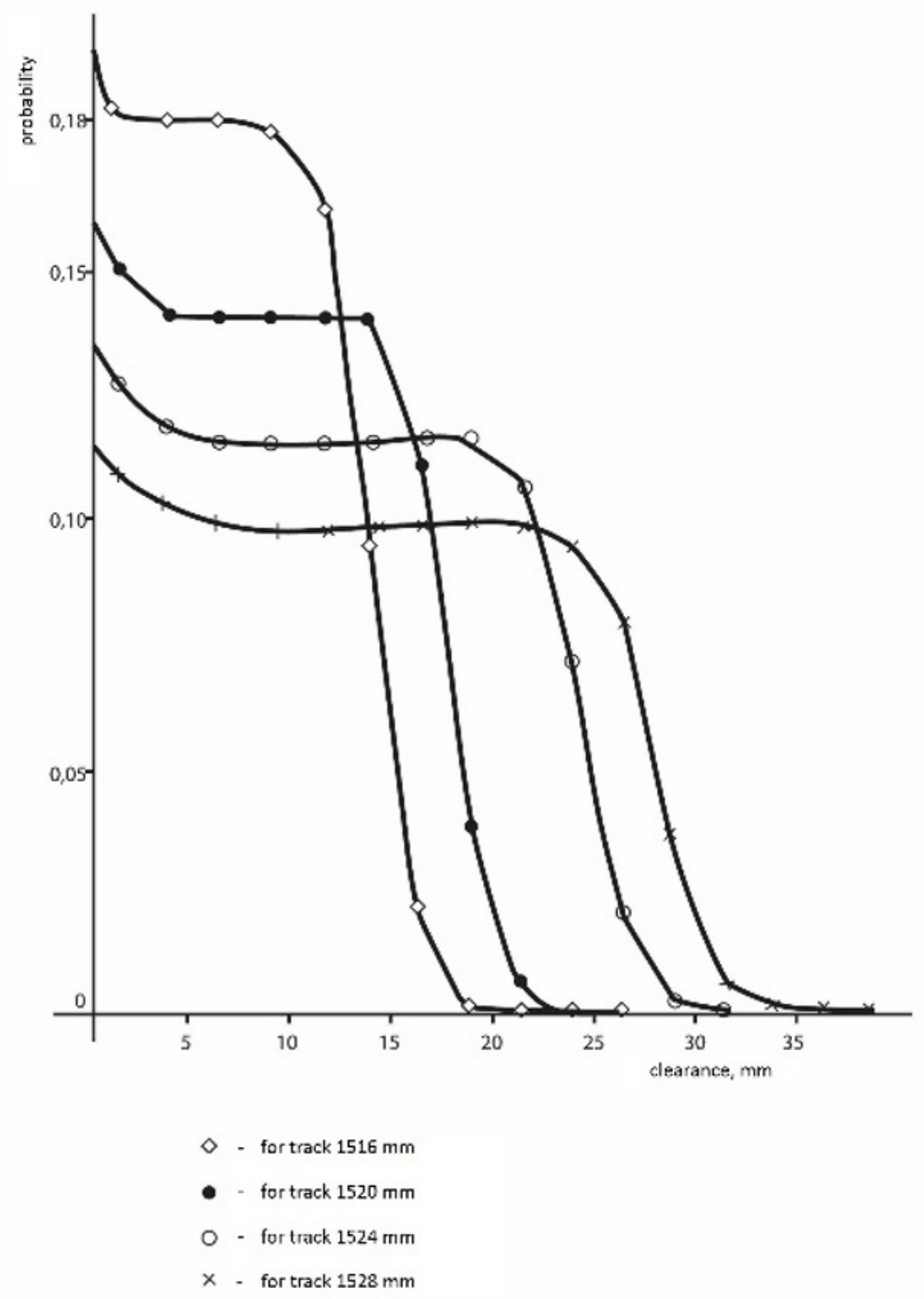

Fig. 1. The probabilities of gaps between the back side of the wheel and the rail at the entrance to the crossbar at the level of measurements 


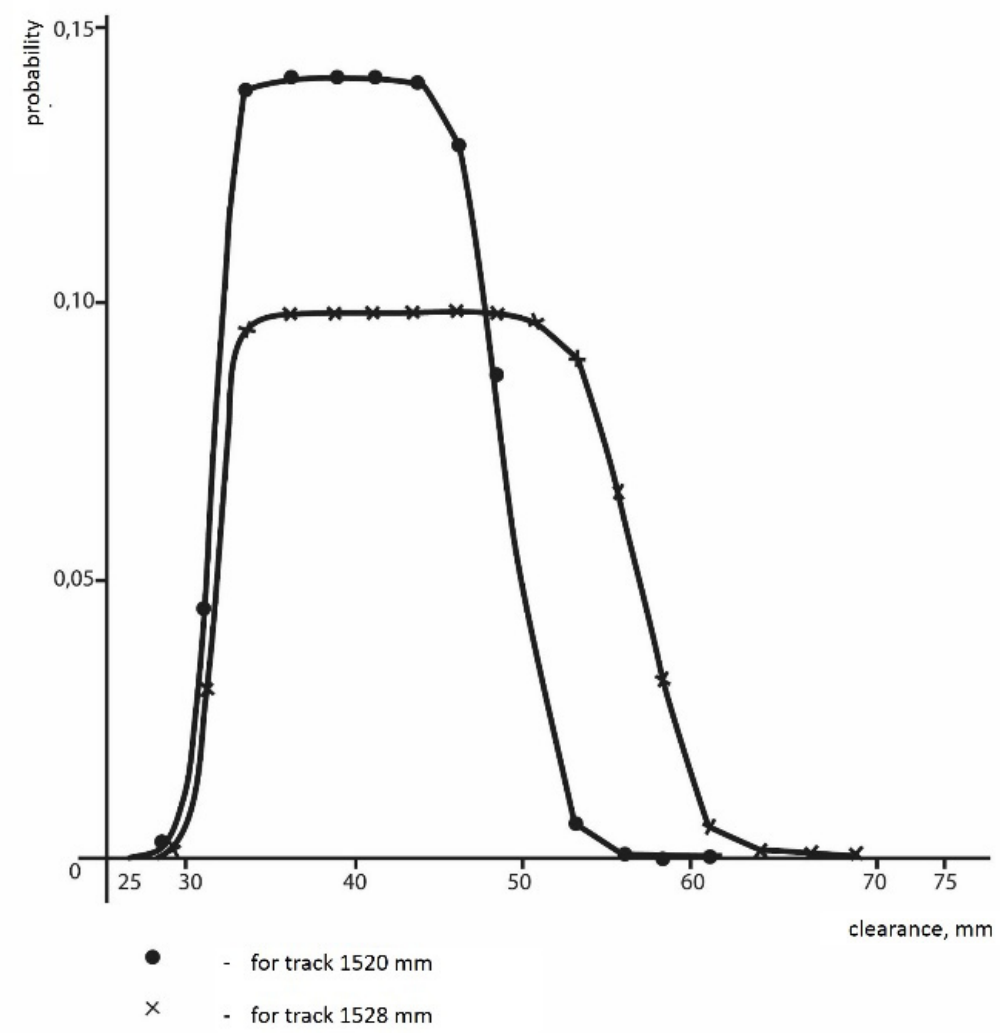

Fig. 2. The probabilities of the values of distances between the working side of the rail at the measurement level and the rear edge of the wheel when entering the crossbar

Before touching the counter-rail of the probability of the position of the rear part of the wheel flange has a distribution $P\left(\delta_{D}\right)$. For those wheels for which $\delta_{D}<e_{\kappa}$, distribution of the gap between the running rail and the back of the ridge after entering the straight part of the counter rail will remain unchanged, since they will not be in contact with the counter rail. Wheels for which $\delta_{D}>e_{\kappa}$ will be in contact with the counter rail. Moving along the counter rail, the wheel causes the elastic deformation of the counter rail $e^{y}$. The position of the wheel in the straight part of the counter rail will be determined by the magnitude of this elastic deformation.

The part of the distribution (Figure 3) that corresponds to the distance between the working surface of the running rail and the non-working surface of the wheel, smaller than the chute of the counter rail, remains unchanged after entering the chute of the counter rail; another part of the distribution is deformed, increasing the probabilities of smaller distances and decreasing the probabilities of larger ones. Moreover, it is clearly seen from Figure 3 that the narrower the groove of the counter rail, the more distorted the distribution is. 


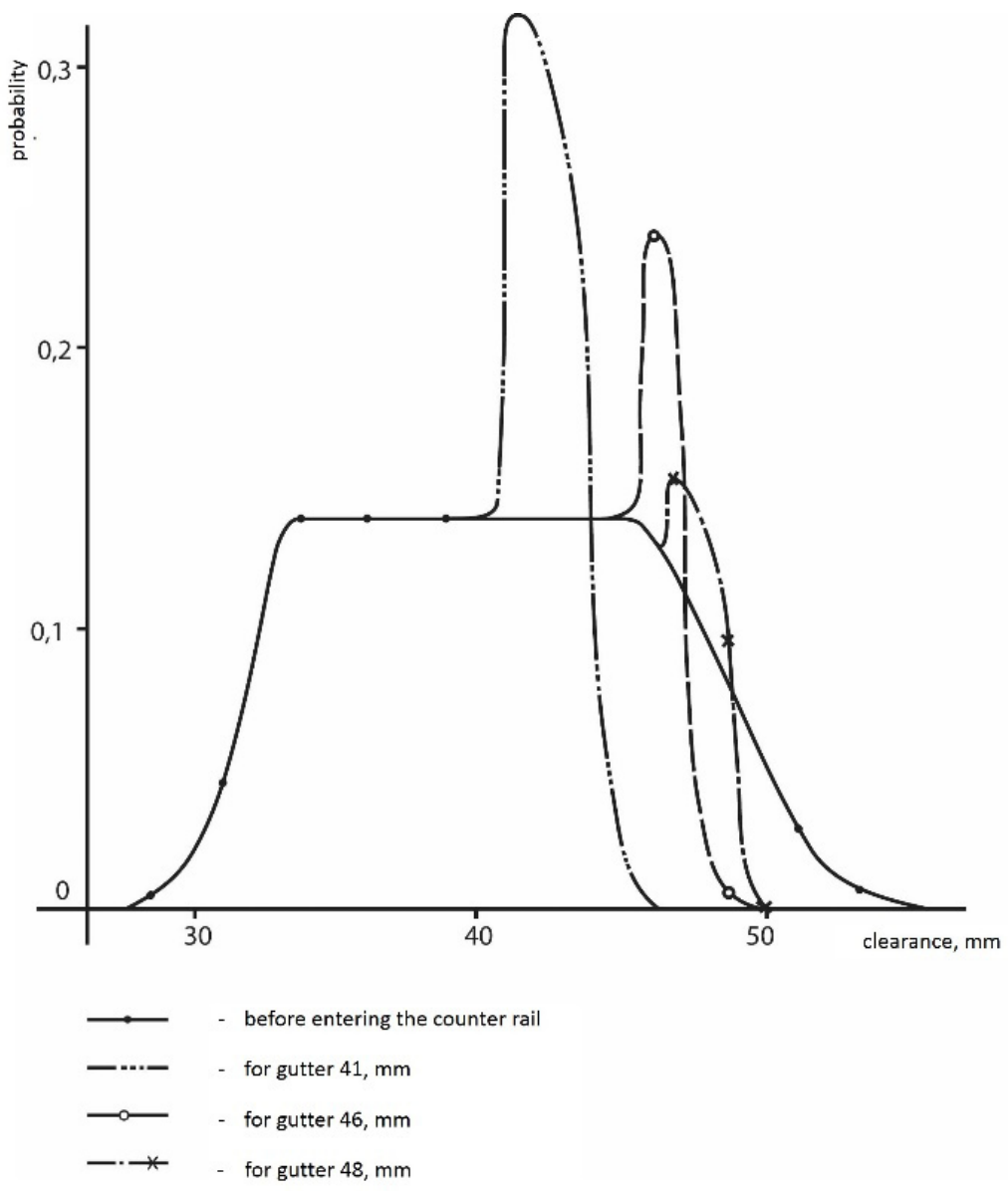

Fig. 3. Values of the distance between the working edge of the rail and the non-working edge of the wheel in the groove of the counter rail for the gauge of $1,520 \mathrm{~mm}$

Table 1 shows the results of a probabilistic calculation of wheel impacts in the catching part of the counter rails.

Table 1. The number of impacts of wheel flanges in the catching part of the counter rails.

\begin{tabular}{|c|c|c|c|c|c|c|c|c|c|c|c|}
\hline Track width, mm & \multicolumn{2}{|c|}{1516} & \multicolumn{3}{|c|}{1520} & & \multicolumn{2}{|c|}{1523} & \multicolumn{3}{|c|}{1524} \\
\hline $\begin{array}{l}\text { Counter rall chute at the end } \\
\text { of the bent portion, mm } \\
\text { Number of impacts* }\end{array}$ & $61-67$ & 61 & $63-67$ & 61 & 63 & 65 & 67 & 61 & 63 & 65 & 67 \\
\hline - in transition to the bent part & 0 & 2 & 0 & 34 & 3 & 0 & 0 & 790 & 14 & 0 & 0 \\
\hline - directly to the catching part & & & & & & & & & & & \\
\hline & 0 & 0 & 0 & 0.7 & 0 & 0 & 0 & 7 & 0 & 0 & 0 \\
\hline
\end{tabular}


* - the number of impacts was calculated on the basis of $21,4 \cdot 10^{6}$ wheel passes, which corresponds to the service life of 300 million tons gross and an average axial load of 140 $\mathrm{kN}$.

From a probabilistic calculation, it can be seen that such strikes can occur only with significant violations of the dimensions of the gauge.

By the condition of preventing the wheels from striking the counter rail at an angle of the catching part, it is advisable to keep the lower limit of tolerance on the counter rail at the beginning of the bent part of $62 \mathrm{~mm}$; the maximum track width can be $1523 \mathrm{~mm}$. This confirms the current standard of tolerance for the width of the gauge in the cross node.

Overrunning of wheels on the bent part of the counter rail occurs at much smaller angles than in the catch part of the counter rail and can be allowed. Probabilistic calculation shows that, in general, about $30 \%$ of the wheels interact with counter rails when passing through the cross joint.

Depending on the specific combinations of geometric dimensions, this percentage can vary from $16 \%$ to $58 \%$ or even more. The level of stresses in the counter rails largely depends on the combination of sizes, therefore the dimensions of the cross-joint should be chosen so as to ensure the strength of the counter rails, that is, the proportion of the impact wheels and the magnitude of the stresses not exceeding the allowable values. By means of the studies, performed during the dynamic strength tests, it was found that with the proportion of wheels hitting the counter rail close to the network average (about 33\%), the allowable stresses in the counter rails can be set to about $330 \mathrm{MPa}$. Calculations of the probability of wheels overrunning on the bent part of the counter rail, some of which are shown in Table 2, and the results of the tests carried out, show that such conditions are met with a track width of $1.523 \mathrm{~mm}$ or less and the existing min. tolerance of $(44-2) \mathrm{mm}$ grooves in the straight part of counter rails $[8,9]$.

Table 2. the probability of wheels overrunning on the bent part of the counter rails.

\begin{tabular}{|c|c|c|c|c|c|c|c|c|c|c|c|c|}
\hline Track width, mm & & & 1520 & & & & 1523 & & & & 1528 & \\
\hline $\begin{array}{l}\text { Trench in the } \\
\text { straight part of } \\
\text { the counter rail, } \\
\mathrm{mm}\end{array}$ & 42 & 44 & 46 & 48 & 42 & 44 & 46 & 48 & 42 & 44 & 46 & 48 \\
\hline $\begin{array}{l}\text { Probabilities and } \\
\text { number of } \\
\text { overrunnings }\end{array}$ & $\begin{array}{l}0.2 \\
7\end{array}$ & $\begin{array}{l}0.1 \\
5\end{array}$ & 0.08 & 0.03 & 0.03 & 0.24 & 0.15 & 0.06 & 0.52 & 0.49 & 0.43 & 0.35 \\
\hline
\end{tabular}

The tests carried out on the way show that for the counter rails, even with unfavorable combinations of gutter size and gauge, in the first half of the bent part, no more than 5-13\% of the wheels come into contact with the counter rail, and the greatest stresses in the counter rail with the covering props of this area do not exceed $50 \div 70 \mathrm{MPa}$. Therefore, the plus tolerance on the size of the gutter at the entrance to the bent part of the counter rail can be increased to $(64+6) \mathrm{mm}$. This makes it possible to recommend to increase the tolerance on the content of the gutter in the catching part of the counter rails at their inputs to $(86+7)$ $\mathrm{mm}$.

Structurally, the counter rails consist of the main part $\boldsymbol{l}_{\boldsymbol{k}}$, overlapping the harmful space, two elbows $\boldsymbol{l}_{\kappa} \boldsymbol{l}$, guide the wheel-sets in the cross of the corresponding track and two nonworking catching parts $\boldsymbol{l}_{\kappa 2}$, designed to ensure traffic safety in cases of violation of the rail track operation norms and in extraordinary cases [10].

The lengths of the parts of the counter rails can be defined as follows: 


$$
\left\{\begin{array}{l}
l_{\kappa o}=\left(t_{2}+w_{c}\right) N+2_{e \kappa}, \\
l_{k 1}=\frac{t_{\kappa 1}-t_{\kappa o}}{\sin \gamma_{\kappa 1}} \\
l_{\kappa 2}=150 \mathrm{~mm}, \\
l_{\kappa}=l_{\kappa o}+2 l_{\kappa 1}+2 l_{\kappa 2}
\end{array}\right.
$$

where $\gamma_{\kappa l}$ - retraction angle of the counter rail; $t_{2}$ - trough in the throat of the cross-joint; $t_{k o}$ - trough in the main part of the cross-joint; $t_{\kappa l}-$ - trough in the end of the bended part of the cross-joint; $w_{c}$ - the width of the cross core of the cross-joint where the wheel rests fully on it $-40 \mathrm{~mm} ; e_{\kappa}$ - stock overlap of the harmful space $-100 \mathrm{~mm}$.

Retraction angle of the counter rail should not exceed the admissible angle of impact in the designated part of the counter rail, which is determined by the permissible value of "strike effect" according to the formula:

$$
\sin \gamma_{k 1}=\frac{W_{\kappa}}{V_{n}}
$$

where $V_{\kappa}$ - estimated speed of traffic on the main way.

High dynamic forces acting on the counter rail, leads to rupture of bolts in joints with counter rails of RK special profiles, which requires to take the immediate measures, up to stop the trains traffic (at break of two bolts in a row). In the joints with counter rails of SP special profiles, increased exposure leads to kinks of counter rail shoes. Wheelset shifts cause the appearance of saddles on the cross-joints within the operation, which requires limiting the speed of the trains and reduces the operational life of the rails themselves [11].

The level of impact of the wheels on the counter-rail is regulated by regulating the values of the "impact effect" on the bent and catching parts of the counter rail. According to the normalized values of the "impact effect", the bend angles of the counter rail in the bent and catching part are determined. These angles, taking into account manufacturing tolerances, are considered in the design documentation for the railway switch.

Before the appearance of counter rails joints that were not connected to the running rail, the counter rails of RK special profiles were used in the construction of switches, which were structurally attached to the rail by inserts. Adjustment of the gutters in this design is extremely difficult and has not been carried out in operation. If the wear on the counter rail did not allow for the required gutter size and safety, the counter rail along with the cross rail should be replaced.

In the process of run-out, the angles of the wheels overrunning on the counter rail practically do not change, therefore the run-out of the counter rails of the RK special profile is not limited by this criterion.

The appearance of counter rail joints with counter rails, not connected with the running rails of the SP special profiles, made it possible to adjust the size of the grooves during operation by installing gaskets between the counter rail and the support part of the counter rail attachments. This makes it possible to extend the service life of the counter rails, since their run-out is no longer an obstacle to the installation of standard values for gutter dimensions and safety.

Due to the fact that the impact of the wheels on the counter rails along their length is not the same, the counter rails do not run-out evenly. The greatest run-out occurs in the middle 
part of the counter rail, therefore, when setting the shims, the angles of the bent part of the counter rail increase. The "impact effect", defined in accordance with formula (11), increases. Table 3 shows the results of calculations of the impact effect when the wheels run over the counter rail for the type P65 switches of the most common brands, with different run-out of the counter rails of the SP special profile. During the calculations, it was assumed that the dimensions of the grooves on the worn-out counter rail were adjusted to values that comply with the current standards for the maintenance of switches.

Table 3. The magnitude of the "impact effect" when the wheels run over the counter rail for switches of the type P65, with different run-out of the counter rails of the SP special profile

\begin{tabular}{clllllllllll}
\hline \multirow{2}{*}{$\begin{array}{l}\text { Mark of the } \\
\text { cross-joint }\end{array}$} & $\begin{array}{l}\text { Construc- } \\
\text { tional speed, }\end{array}$ & \multicolumn{10}{c}{ Counter rail run-out, mm } \\
\cline { 2 - 12 } & $\mathrm{km} / \mathrm{h}$ & 0 & 2 & 4 & 6 & 8 & 10 & 12 & 14 & 16 \\
\hline \multirow{3}{*}{$1 / 11$} & 140(straight) & 0.53 & 0.57 & 0.63 & 0.68 & 0.74 & 0.79 & 0.84 & 0.90 & 0.96 \\
& 50(side) & 0.19 & 0.21 & 0.23 & 0.25 & 0.27 & 0.29 & 0.30 & 0.32 & 0.34 \\
& 100(straight) & 0.57 & 0.63 & 0.69 & 0.75 & 0.80 & 0.86 & 0.92 & 0.98 & 1.03 \\
$1 / 9$ & 40(side) & 0.23 & 0.25 & 0.28 & 0.30 & 0.32 & 0.35 & 0.37 & 0.39 & 0.41 \\
\hline
\end{tabular}

The practice of designing of cross-shaped switches used in the USSR, and then in Russia, the magnitude of the "impact effect" when wheels hit the counter rail elements is limited to $\mathrm{W}=(0.4-0.6) \mathrm{m} / \mathrm{s}$, at nominal sizes [12]. With dimensions corresponding to the maximum adverse combinations, this value is $\mathrm{W}=0.8 \mathrm{~m} / \mathrm{s}$. The adjustment of the gutters with gaskets on the counter rail joints with counter rails of the SP special profile is similar to the formation of such conditions, therefore the value limiting the maximum value of the "impact effect" should be considered as $\mathrm{W}=0.8 \mathrm{~m} / \mathrm{s}$.

The complexity of the design of the counter-rail joints makes it difficult to analytically study the stress-strain state of the counter rails, therefore, in this work, the experimentallycalculated method is used to study the stress-strain state of the counter rails.

The first stage of this part of the work is dynamic strength tests with the study of the parameters of the stress-strain state of the counter rails along the way.

The third way of the Experimental ring of JSC VNIIZhT was chosen as a pilot site. As an experimental train was used the train circulating along the ring with the axial load of half-cars up to $27 \mathrm{tf} /$ axis. The movement was carried out at speeds up to $80 \mathrm{~km} / \mathrm{h}$.

The comparison showed that there is no fundamental difference in the stress distribution of the counter rails of different structures (SP-850 and PK65) in the limiting sections. The maximum principal normal stresses occur on the upper lateral edge of the counter rail of the special profile PK65 at the beginning of the bend and reach $330 \mathrm{MPa}$, the counter rail from the corner of the counter rail SP-850, they are $300 \mathrm{MPa}$.

As a result of the comparative analysis of work in the way of the RC65 and SP-850 counter rails, it can be concluded that, despite the decrease in the metal intensity of the counter rail from the corner of the rail SP-850, due to a more rational distribution of the metal over the cross section, the level of stresses in it not only haven't increased, but decreased by an average of $8 \%$, and at limiting points by $23 \%$, which confirms the advisability of using the SP-850 special profile instead of the RK65 special profile. However, the excessive rigidity of the counter rail itself and its fastening in the horizontal transverse direction, confirmed by comparing the data on the wringing out of the elements of the cross joint, confirms data on a higher intensity of its wear-out.

Studies of the stress-strain state of the counter rails with different wear-out were performed using a calculation method. The finite element method and the ANSYS software package that implements it were used. 
For the calculations, a model of a half counter rail was developed, which is a 4-span, supported on 5 pillars.

Load value of $0.1 ; 0.2 ; 0.6$ and $0.9 \cdot \mathrm{P}$ is applied to the spans between the supports in order to simulate the process of the counter rail operation under the train load.

Simulation was carried out for loads up to $\mathrm{P}=150 \mathrm{kN}$.

The results of calculations for the counter rail of the SP-850 special profile are illustrated in Figure 4, and the results of processing the calculation materials are presented in Table 4.

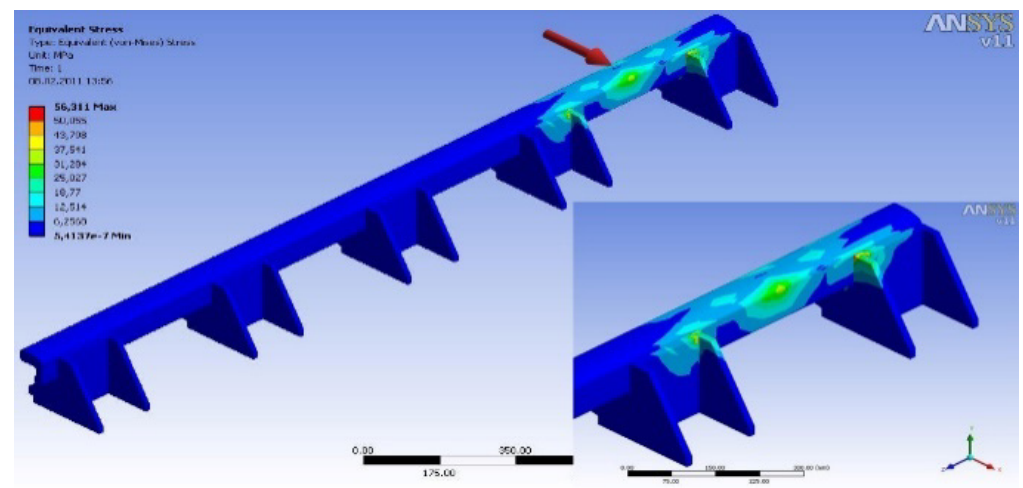

Fig. 4. Results of SP-850 simulation for the load in the middle part of the counter rail

Table 4. The highest values of stresses in the spans between the supports of the Russian Standard SP-850 rail, MPa

\begin{tabular}{ccccc}
\hline The highest & \multicolumn{4}{c}{ Span numbers (from the middle of the counter rail) } \\
\cline { 2 - 4 } stresses, MPa & $0-1$ & $1-2$ & $2-3$ & $3-4$ \\
SP-850 & 265 & 252 & 163 & 56 \\
\hline
\end{tabular}

The adequacy of the model was checked by comparing the stress values obtained from the calculations with the results of dynamic strength tests. The obtained values are close (the difference of the maximum values is $13 \%$ ), that is the model adequately reflects the stress state of the counter rails of the SP-850 special profile. This makes it possible to investigate on this model the change in the stress-strain state of the counter rails when they are differently worn-out and to determine the limiting value of counter rail wear-out by criterion of its durability.

Changes of sections were put in the model and variant calculations were carried out.

Changing the geometry of the model accordingly and performing the calculations, we obtain the data given in Table 5.

Table 5. Change of the highest stresses in the counter rail of the special profile in the process of run-out.

\begin{tabular}{|c|c|c|c|c|c|c|c|c|c|}
\hline \multirow{2}{*}{$\begin{array}{l}\text { Characteristic of the stress } \\
\text { state }\end{array}$} & \multicolumn{9}{|c|}{ Counter rail run-out, $\mathrm{mm}$} \\
\hline & 0 & 2 & 4 & 6 & 8 & 10 & 12 & 14 & 16 \\
\hline $\begin{array}{l}\text { The increase in stress at the } \\
\text { most loaded point of the } \\
\text { counter rail, times }\end{array}$ & 1.00 & 1.02 & 1.04 & 1.08 & 1.11 & 1.14 & 1.20 & 1.25 & 1.30 \\
\hline
\end{tabular}

\section{Results of the researches}

Analysis of the data obtained shows that under the terms of the non-exceedance of the "impact effect" in the bent part of the counter rail, the largest values of run-out for the direct way counter rails of the SP special profiles should not exceed $10 \mathrm{~mm}$ for switches of 
mark $1 / 11$ and $8 \mathrm{~mm}$ for switches of mark 1/9. Additionally, it should be noted that such conditions of overrunning are realized when the wheels are running on the catching part of more than $3 \mathrm{~mm}$.

Thus, according to the criterion of the "impact effect" the limit wear-out of the counter rails from the SP 850 special profile for the direct way of switches should be $13 \mathrm{~mm}$ for switches of the mark $1 / 11$ and $11 \mathrm{~mm}$ for switches of mark 1/9.

The wear-out of the sidetrack counter rails is not limited by this criterion.

If we proceed from the currently established allowable stress value in the counter-rail $330 \mathrm{MPa}$, then the highest allowable stress increase factor is 330: $265=1.25$ times.

Consequently, in terms of the strength parameter, the maximum allowable wear-out of the counter rails of the SP 850 special profile is $14 \mathrm{~mm}$.

\section{References}

1. Loktev, A.A., Korolev, V.V., Poddaeva, O.I., Stepanov, K.D., Chernikov, I.Y Vestnik of the Railway Research Institute. 77(2), pp. 77-83 (In Russian) (2018). https://doi.org/10.21780/2223-9731-2018-77-2-77-83

2. Belošević, I., Ivić, M. et al.: Challenges in the Railway Yards Layout Designing Regarding the Implementation of Intermodal technologies. In: 2nd Logistics International Conference. Aćimović, pp. 62-67 (2015).

3. Li Shen, Xiukun Wei, Limin Jia: Surface defects detection of railway turnouts, 34th Chinese Control Conference (CCC) (2015). DOI: 10.1109/ChiCC.2015.7260626

4. Mateusz, F., Woloszyn, M.: Energy efficiency analysis of railway turnout heating with a simplified snow model using classical and contactless heating method, 2018 International Interdisciplinary PhD Workshop (IIPhDW) (2018). DOI: 10.1109/IIPHDW.2018.8388237

5. Belošević, I., Milinković, S. et al.: A Fuzzy Group Decision Making for a Rail-Road Transshipment Yard Micro Location Problem. In: MATEC Web of Conferences, vol. 235, paper 00019 (2018).

6. Glusberg, B., Korolev, V., Shishkina, I., Loktev, A., Shukurov, J., Geluh, P.: MATEC Web of Conferences, vol. 239, 01054 (2018). doi.org/10.1051/matecconf/201823901054 DOI: $10.1051 /$ matecconf $/ 201823901054$

7. Loktev, A. A., Korolev, V. V., Shishkina, I. V.: IOP Conf. Series: Materials Science and Engineering, 463 (2018). 032019 doi:10.1088/1757-899X/463/3/032019

8. Milosavljević, M., Bursać, M. et al.: Selection of the railroad container terminal in Serbia based on multi-criteria decision-making methods. Decision Making: Applications in Management and Engineering, vol. 1, pp. 1-15 (2018).

9. Savin, A., Kogan, A., Loktev, A., Korolev, V.: Journal of Innovative Technology and Exploring Engineering (IJITEE) ISSN: 2278-3075, vol. 8 (2019). G5991058719/19@BEIESP

10. Glusberg, B., Savin, A., Loktev, A., Korolev, V., Shishkina, I., Alexandrova, D., Loktev, D.: Advances in Intelligent Systems and Computing, vol 982, pp. 556-570 (2020). https://doi.org/10.1007/978-3-030-19756-8_53

11. Glusberg, B., Loktev, A., Korolev, V., Shishkina, I., Alexandrova, D., Koloskov, D.: Advances in Intelligent Systems and Computing, vol. 982, pp. 337-345 (2020). https://doi.org/10.1007/978-3-030-19756-8_31

12. Loktev, A., Korolev, V., Shishkina, I., Chernova, L., Geluh, P., Savin, A., Loktev, D., Advances in Intelligent Systems and Computing, vol. 982, pp. 325-336 https://doi.org/10.1007/978-3-03019756-8_30. 